\title{
Somos todos iguais virtualmente? analisando os moderadores idade, gênero e experiência no uso do facebook
}

\author{
Virtually are we all equal? analyzing the moderators age, gender and \\ experience in the use of the facebook
}

\author{
Monize Sâmara Visentini ${ }^{1}$ \\ Daiane Lindner Radons ${ }^{2}$ \\ Fernanda Bard Chagas ${ }^{3}$
}

\begin{abstract}
Resumo
Esta pesquisa identifica a percepção de usuários brasileiros com relação aos motivadores do uso do Facebook, além de analisar como os moderadores "idade", "gênero" e "experiência de uso" na rede social influenciam essa percepção. A revisão da literatura aborda a avaliação das variáveis "idade", "gênero" e "experiência de uso" de redes sociais. Aplicou-se um levantamento com454 usuários do Facebook. Os resultados indicam que o Facebook é uma plataforma de fácil uso, mas que não incita imersão por parte de seus usuários. Observou-se que o gênero feminino apresenta maior intenção de uso da rede social quando esta se mostra de fácil uso. Com relação à idade, destaca-se a associação entre jovens e a facilidade para aprender e utilizar o Facebook.
\end{abstract}

Palavras-chave: Facebook. Intenção Comportamental de Uso. Redes Sociais Virtuais.

\begin{abstract}
This research identifies the perception of Brazilian users regarding the motivators of use of Facebook, as well as analyzes how perception is influenced by the moderators age, gender and user experience on the social network. The literature review addresses the evaluation of the variables age, gender and experience of use in social networks. Was applied a survey to 454 users from Facebook. The results indicate that Facebook has a platform that is easy to use, but does not encourage immersion by its users. It was observed that the female gender shows greater intention to use the social network when it easy to use. With regard to age, there is an association between young people and the facilitation in learning and Facebook.
\end{abstract}

Keywords: Facebook. Intentional Behavior of Use. Virtual Social Networks.

\section{Introdução}

As redes sociais virtuais são ferramentas de comunicação e informação que dispõem de plataformas de fácil acesso e uso. Geralmente, essas redes permitem aos seus usuários a oportunidade de obter informações em tempo integral, além de expandir e fortalecer círculos de interações (SHIMAZAKI; PINTO, 2011). Nos últimos anos, elas têm se tornado essenciais nas mais variadas atividades, desde comerciais e informativas até de cunho instrutivo e educacional. Parte disso se deve principalmente ao poder de influência que essas redes representam para a comunicação, uma vez que possuem um espaço que proporciona maior liberdade para as interações (ROCHA; CHRISTOPOULOS, 2013).

Doutora em Administração pela Universidade Federal do Rio Grande do Sul

Professora do Curso de Administração e do Programa de Pós-Graduação em Desenvolvimento e Políticas Públicas da Universidade Federal da Fronteira Sul. Brasil. Afiliação:

Universidade Federal da Fronteira Sul. Lattes: http://lattes.cnpq.br/6482101738421142 Email: monize.visentini@uffs.edu.br

2 Mestre em Administração pela Universidade Federal de Santa Maria. Assistente em Administração na Universidade Federal da Fronteira Sul. Brasil. Afiliação: Universidade Federal da Fronteira Sul. Lattes: http://lattes.cnpq.br/8068136456971738 Email: daialindner@gmail.com

3 Graduação em Administração pela Universidade Federal da Fronteira Sul. Brasil. Afiliação: Universidade Federal da Fronteira Sul. Lattes: http:// lattes.cnpq.br/1690983751071410 Email: fernandabard@hotmail.com 
O engajamento crescente nas redes sociais tem repercutido na comunidade acadêmica, que, ao longo dos últimos anos, explora as motivações de uso e suas diversas aplicabilidades.No cenário nacional, Löbler, Visentini e Estivalete (2011) seguiram o modelo de Agarwal e Karahanna (2000), identificando como a absorção cognitiva motivava a intenção de uso dos usuários do Orkut. A absorção cognitiva é compreendida como o estado de profundo envolvimento com a tarefa individual que está sendo executada com o apoio da tecnologia da informação, consistindo num fator pessoal que contribui para o entendimento das intenções individuais e o uso da informação tecnológica (GALLUCH; THATCHER, 2006). Os autores também verificaram que a intenção do uso dos usuários era explicada por $41 \%$ da absorção cognitiva, e que o fator curiosidade possuía relevância significativa para o uso dessa rede social.

Visentini et al. (2016) desenvolveram um modelo integrado que analisa as relações entre alguns fatores comportamentais e a intenção comportamental de uso de usuários brasileiros no Facebook. Os autores levam em consideração os fatores "utilidade no trabalho", "utilidade informacional", "facilidade de uso percebida", "imersão focada" e "entretenimento", baseando-se nos modelos de Agarwal e Karahanna (2000), Lin e Lu (2011), Verhagen et al. (2012) e Venkatesh et al. (2003). Ao fim, demonstrou-se que a "intenção de uso" do Facebook é impactada principalmente pela "utilidade informacional", seguida das influências dos constructos "entretenimento", "utilidade no trabalho" e "facilidade de uso percebida". Souza, Filenga e Sanchez (2011) utilizaram a Teoria Unificada de Aceitação e Uso de Tecnologia - UTAUT (Unified Theory of Acceptance and Use of Technology) de Venkatesh et al. (2003), para identificar o que levava os usuários das redes sociais Facebook, Orkut e Twitter a adotarem a tecnologia. Concluíram que o fator 'influência social'impactava de modo significativo no uso desses canais de comunicação. Moraes, Cappellozza e Meirelles (2014), ao buscarem identificar a intenção dos usuários de se engajarem em protestos pelo Facebook, também basearam seu estudo nos fatores apresentados por Venkatesh et al. (2003), analisando os moderadores "idade", "experiência", "gênero" e "voluntariedade". Os resultados indicaram que a "expectativa de esforço" influenciava positivamente as manifestações nas redes sociais. $O$ teste com esses moderadores para complementar futuras pesquisas também é sugerido pelo trabalho de Souza, Filenga e Sanchez (2011).

Conforme Venkatesh, Thong e Xu (2012), os fatores moderadores são variáveis próprias e que diferem em cada indivíduo, podendo interferir diretamente nos fatores que impactam na aceitação e no uso da tecnologia e, por isso, devem ser estudados. Conforme esses autores, os fatores que motivam o usuário a utilizar a tecnologia estão relacionados às condições facilitadoras, mediadas pelos moderadores "idade", "gênero" e "experiência". Nesse sentido, Liao (2015) atenta para as diferenças na utilização da tecnologia por parte dos idosos em comparação aos usuários mais jovens, uma vez que os primeiros tendem a possuir maior dificuldade para adaptar-se em plataformas virtuais que apresentam certo grau de complexidade. Morris e Venkatesh (2000) constataram que as habilidades mentais humanas se alteram ao longo do tempo, tornando-se interessante investigar a influência da faixa etária na utilização das redes sociais virtuais. Corroborando, Venkatesh, Xong e Xu (2012) discorrem acerca da idade no uso da tecnologia, atestando que usuários mais velhos enfrentam maiores dificuldades em processar novos conteúdos, o que afeta a sua disposição em aprender a usar essas novas tecnologias.

Os usuários também possuem diferenças significativas com relação ao gênero, demonstrando que homens utilizam as redes sociais com intuitos diferentes das mulheres, e também interagem de formas distintas, ratificando as especificidades psicológicas e comportamentais inerentes a cada gênero. $O$ gênero masculino e o feminino percebem a utilidade da tecnologia de formas discrepantes (TARHINI; HONE; LIU, 2014):enquanto o primeiro está voltado para a realização de suas tarefas, o segundo procura utilidades hedônicas (SUN et al., 2015). Isso pode estar relacionado ao fato de os gêneros serem orientados pela sociedade de formas diferentes: os homens são mais direcionados para tarefas e atividades, e as mulheres, para o consumo e o entretenimento (VENKATESH; XONG; XU, 2012).

A "experiência de uso" é o moderador que reflete as habilidades ou conhecimentos adquiridos conforme um determinado tempo de uso da tecnologia passa. Os hábitos também são definidos pela experiência, ou seja, quanto mais tempo a pessoa utiliza uma determinada tecnologia, mais familiar elase torna, fazendo com que o indivíduo desenvolva diferentes habilidades (VENKATESH, THONG, XU, 2012). 
Venkatesh et al. (2003) indicam que o moderador "experiência de uso" influencia a utilidade percebida pelos usuários da tecnologia, sendo que a utilidade aumenta conforme a experiência.O fator "facilidade de uso", contudo, se torna insignificante conforme a experiência aumenta.

Na literatura internacional, nota-se uma tendência de publicações que analisam os fatores moderadores da motivação de uso das redes sociais virtuais e de seus consequentes antecedentes,entretanto esta perspectiva de investigação no contexto nacional ainda é incipiente. Tendo em vista a necessidade de aprofundar estudos que compreendam o comportamento de uso de redes sociais virtuais, a seguinte questão de pesquisa é considerada: Como os usuários do Facebook percebem os fatores que contribuem para a utilização dessa plataforma e de que forma moderadores interferem nessa relação?Assim, partindo da proposição das variáveis e dos fatores influentes no uso das redes sociais virtuais apontados por Visentini et al. (2016), este trabalho tem como objetivo geral investigar a percepção de usuários brasileiros com relação aos motivadores do uso do Facebook.Adicionalmente, visa-se analisar de que modo os moderadores "idade", "gênero" e "experiência de uso" na rede social influenciam nessa percepção.

A seleção do Facebook como rede social de investigação decorre do fatoda rede também ter sido a analisada no trabalho de Visentini et al. (2016), bem como pela sua relevância no contexto digital. Conforme dados da Comscore (2014), o Brasil lidera o ranking de países com maior crescimento em número de usuários do Facebook, chegando a ter $94 \%$ dos internautas brasileiros ativos em 2013. Entende-se que as redes sociais virtuais possibilitam novas formas de realizar negócios e que,sendo assim, compreender como os usuários percebem as características influentes no uso, bem como o que modera tais características, pode contribuir para que as organizações adaptem seus canais de comunicação com os clientes e/ou desenvolvam ferramentas inovadoras de relacionamento.

\section{Revisão de Literatura: Moderadores de uso de redes sociais}

\subsection{Idade}

A "idade" é um dos fatores moderadores da percepção do usuário em relação à tecnologia, conforme achados de pesquisadores como Morris e Venkatesh (2000), os quais exploram aspectos da faixa etária ao compararem dois grupos de trabalhadores em função de suas idades e constatam que as decisões de uso da tecnologia dos trabalhadores mais jovens estão ligadas à sua intenção de utilizar a tecnologia, enquanto que, para os mais velhos, essas decisões estão associadas às novas normas da empresa, reforçando a premissa de que usuários de maior idade estão menos propensos ao uso da nova tecnologia. Elias, Smith e Barney (2012) também analisam a intenção de utilizar a tecnologia conforme a idade dos colaboradores de uma organização e verificam que alguns usuários idosos, quando não satisfeitos com o trabalho, ou quando o grau de dificuldade do sistema é alto, não possuem intenção de utilizar o novo sistema.

Tarhini, Heno e Lin (2014) reiteram isso ao concluir que a "facilidade de uso" é afetada pela faixa etária quando os usuários demonstram idade mais avançada, indicando que a tecnologia torna-se mais atraente quando a plataforma é de fácil interação. A "percepção de utilidade" da tecnologia é maior para os usuários mais novos, e influencia a sua intenção de uso da ferramenta.Seebaluck et al. (2015) atestam que o fator "facilidade de uso" é o responsável pelo menor número de adultos em idade avançada dentro das redes sociais virtuais, de forma que a categoria de jovens computa maior número de usuários dessas redes. Apesar de a facilidade auxiliar o maior número de jovens nesses canais, também se evidenciou que encontram maior utilidade para as redes virtuais que os adultos. Labib e Mostafa (2015), ao estudarem o uso desses canais por jovens que ainda estavam na graduação e adultos que já haviam se graduado, verificaram que, para os jovens, existe um propósito educacional e de aprendizado no uso das redes sociais, enquanto que os asultosas usam com fins de socialização e entretenimento na maior parte dos casos.

Pfeil, Arjan e Zaphiris (2009) analisaram usuários do MySpace, comparando dois grupos: um grupo de usuários com mais de 60 anos e outro com pessoas de idades entre 13 a 19 anos. Foi identificado que as diferentes faixas etárias afetam o modo como os dois grupos interagem na rede social, sendo os jovens mais propensos a estabelecerem amizade com grupos de idade semelhante e a interagirem nas redes com vários usuários, tendo um número abrangente de amigos ou seguidores nesses canais de relacionamento, 
enquanto os mais velhos possuem uma rede de amizades pequena, porém diversa no que tange à idade, tendo amigos tanto jovens quando idosos ou de meia idade.

Ainda sobre as redes de amizade, Nosko, Wood e Molema (2010) evidenciaram que, quanto mais velho o usuário for, menos propenso é para revelar informações pessoais nas redes de relacionamento e tende a apresentar restrição em relação ao seu grupo de amizades, optando por interagir com pessoas conhecidas. Corroborando, Ji et al. (2014) concluem que os jovens chineses se sentem mais à vontade para revelar informações pessoais nas redes sociais do que os mais velhos. Isso se dá principalmente pela percepção dos jovens de que os benefícios das redes virtuais são mais impactantes do que os malefícios de ter sua imagem exposta globalmente. Falahah (2012) reitera que os jovens não possuem a ansiedade e experiência dos adultos para refrearem seu uso indiscreto das redes sociais.

\subsection{Gênero}

Diversos autores (VENKATESH, MORRIS, 2000; MARUPING, MAGNI, 2012; SUN et al, 2015; TARHINI, HONE, LIU, 2014) estudaram a influência do moderador "gênero" na intenção de utilizar tecnologias. Venkatesh e Morris (2000) constataram que a utilização da tecnologia por homens estava diretamente relacionada ao constructo "utilidade percebida", enquanto que, para as mulheres, essa relação estava estabelecida pela "percepção de facilidade de uso" e pelas normas da empresa. Maruping e Magni (2012) identificaram que homens, quando em equipe, possuem maior preponderância que as mulheres para explorar a tecnologia ou o novo sistema.

Ao estudar a diferença na percepção dos gêneros, Sun et al. (2015) e Tarhini, Hone e Liu (2014) verificaram que os homens tendem a prestar mais atenção na utilidade das redes sociais, enquanto as mulheres são mais atentas aos benefícios hedônicos, como entretenimento e prazer em utilizar tecnologia. Além disso, para as mulheres, o uso da tecnologia pode estar relacionado à facilidade de uso (TARHINI; HONE; LIU, 2014). No entanto, o estudo de Kavoura e Stavrianea (2015) vai de encontro com esses achados, ao identificar que as mulheres possuem uma linguagem mais profissional e objetiva nas redes de relacionamento virtual, e que procuram comentários acerca de negócios e produtos acima de tudo, buscando a opinião e a experiência de outros consumidores.

Muscanell e Guadagno (2012) identificaram como o gênero dos usuários e suas personalidades afetam a utilização das redes de relacionamento por meio da aplicação de uma escala de personalidade chamada Big Five. Concluíram que, enquanto as mulheres usavam o Facebook e o MySpace para manter relacionamentos já existentes, os homens utilizavam com o intuito de começar novas amizades e encontrar novas pessoas. Corroborando com esses achados, Sheldon (2015), ao verificar a influência de gênero para adicionar colegas e professores no Facebook, identificou que as mulheres são mais cautelosas quanto à interação aluna-professor nas redes sociais do que os homens. O gênero feminino também mostrou maior discrição quanto ao compartilhamento de informações pessoais (JI et al., 2014). Kavoura e Stavrianea (2015), ao compararem o gênero masculino com o feminino no uso das redes sociais, identificaram que, para as mulheres, há menos necessidade de pertencer a um grupo ou sentir aprovação social de suas imagens, enquanto o contrário foi identificado para os homens. Can e Kaya (2016) também deparam com a figura feminina mais presente em grupos de negócios nas redes sociais, onde o principal objetivo é o debate de experiências e a divulgação de propagandas e adds nas redes sociais virtuais.

Para Venkatesh, Thong e Xu (2012), o fator moderador "gênero" apresenta discrepâncias quando associado também à "idade", impactando de forma diferente na "facilidade de uso" e na "utilidade percebida" da tecnologia.Como exemplo pode-se citar que, para mulheres mais velhas, a facilidade de uso é mais importante nas primeiras etapas de uso da tecnologia, e os homens mais jovens tendem a exibir maior procura por inovação e novidades. A percepção da tecnologia entre o gênero feminino e o masculino apresenta diferenças apenas entre usuários mais velhos, enquanto que, para os usuários jovens, essas percepções são tidas como homogêneas (MORRIS; VENKATESH; ARCKERMAN, 2005).

Evidencia-se que cada gênero possui suas especificidades por pertencer a um grupo social, conforme observam Correa, Pinto e Batinga (2016) ao reforçarem em seu estudo a ideia de que cada grupo social tem suas formas de (re)construção e manipulação dos significados. 


\subsection{Experiência de uso}

A "experiência de uso" é definida, segundo Venkatesh, Thong e Xu (2012), como o tempo em que o usuário utiliza a tecnologia, considerando que, conforme a experiência aumenta, o usuário cria maior familiaridade com o uso da inovação. ao familiarizar-se com a tecnologia, entretanto, o usuário tende a considerar alguns fatores como obsoletos, como é o caso da "facilidade de uso", dos "fatores sociais" e das "normas subjetivas" (KARAHANNA; STRAUB; CHERVANY,1999). Esses autores identificaram que as decisões de uso de um sistema ou tecnologia tornam-se mais fáceis e importantes conforme aumenta a experiência do indivíduo, enquanto que as decisões de uso relacionadas com as normas da empresa tornam-se insignificantes. Ademais, Omar, Dahalan e Yusoff (2016) reiteram que o uso de redes virtuais em empresas é identificado por colaboradores como facilitadoras da comunicação, especialmente para aqueles que estão na empresa e as usam há mais de cinco anos, de forma que ratificam que a experiência dos usuários auxilia na percepção de benefícios no uso da nova tecnologia.

Considerando a facilidade e a intenção de uso, Szajna (1996) identificou em um sistema de correios eletrônicos que a primeira (facilidade) não apresentava diferenças significativas quando a experiência de uso dos usuários aumentava. Já Hart, Sutcliffe e Angeli (2013) identificaram que, conforme o tempo de uso e a experiência de navegação em websites crescem, os usuários passam a se familiarizar com os aspectos estruturais das páginas, afetando positivamente a intenção de utilizá-las.

Park, Lee e Kim (2012) estudaram a relação entre as personalidades dos usuários e os padrões de uso das redes sociais. Como resultado identificaram que o tempo gasto no Facebook está direta e positivamente ligado à quantidade e à variedade de amigos no canal de relacionamento. Ryan e Xenos (2011), ao desenvolverem um levantamento com 1.158 usuários do Facebook, identificaram que o tempo gasto na rede social está associado aos traços da personalidade, como a timidez, a solidão e o narcisismo. Usuários tímidos podem encontrar nas redes de relacionamento uma oportunidade de se comunicarem sem restrições e com maior liberdade do que o fazem na vida offline. Já os usuários narcisistas percebem nas redes sociais a chance de expor seus perfis e fotos, no intuito de serem reconhecidos afetivamente.

Correa, Hinsley e Zuniga (2010) realizam cruzamentos de dados de gênero e personalidade a fim de identificar a influência no tempo de uso das redes sociais. Verificaram que ambos, homens e mulheres extrovertidos, tendem a dedicar um maior tempo no canal de comunicação. Também foi identificado que homens com maior instabilidade emocional estão mais propensos a permanecerem por mais tempo nas redes sociais virtuais. Pantano e Gandini (2017) também verificaram que, entre os jovens, os mais extrovertidos e menos individualistas possuem preponderância em adquirir experiência no uso das redes sociais, ainda mais quando visualizam alguma utilidade nesses canais. Ademais, esses jovens criam laços de confiança com a plataforma conforme passam a usá-la diariamente.

\section{Método do Estudo}

Realizou-se pesquisa descritiva, de caráter quantitativo, ao identificar e caracterizar o comportamento dos pesquisados. Como estratégia de pesquisa, foi realizado um levantamento. Conforme Baker (2001), o levantamento permite descobrir fatos, determinar atitudes e opiniões, assim como contribui para o entendimento de comportamentos, utilizando-se de avaliação, análise e descrição de uma população baseada em uma amostra. Nesse sentido, questionários foram aplicados junto a acadêmicos de duas instituições federais de ensino superior do estado do Rio Grande do Sul, uma localizada na região central e outra na Região das Missões, durante o primeiro semestre de 2016. Uma amostra não probabilística de usuários da rede social Facebook foi selecionada e, ao final do processo de coleta de dados, obteve-se 454 questionários válidos.

A amostra deste estudo é composta por 454 estudantes, sendo 282 (62\%) do gênero feminino e 172 (38\%) do gênero masculino. Em relação à idade, 264 pessoas possuem 20 anos ou menos, e 190 pessoas têm 21 anos ou mais. O curso de graduação predominante na amostra foi Administração $(n=130)$, seguido 
de Agronomia ( $n=95)$, Engenharia Ambiental $(n=51)$, Psicologia $(n=45)$ e Relações Públicas $(n=42)$, entre outros cursos. A experiência de uso dos respondentes foi considerada a partir do tempo de acesso diário ao Facebook e verificou-se que a maior parte dos acadêmicos $(56 \%)$ acessa diariamente a rede social por 2,44 horas ou menos $(n=254)$, enquanto que aqueles que utilizam 2,45 horas ou mais $(n=200)$ foram responsáveis por $44 \%$ das respostas. O corte de 2,44 horas corresponde ao valor médio de horas de acesso dos respondentes da pesquisa ao Facebook, sendo definido como o balizador para a definição arbitrária dos usuários mais ou menos experientes.

Para a coleta de dados, utilizou-se como base o instrumento de Visentini et al. (2016), a fim de mensurar os fatores motivadores do uso das redes sociais levando em consideração a "utilidade informacional", a "utilidade do trabalho", a "facilidade percebida", a "imersão focada", o "entretenimento" e a "intenção comportamental de uso"das redes sociais virtuais. Para medir esses fatores, 21 variáveis (Tabela 1) foram incluídas no questionário, utilizando uma escala tipo Likert, variando entre discordo totalmente (1) e concordo totalmente (7).

Também foram realizados questionamentos em relação ao perfil do respondente, como idade, gênero, curso de graduação frequentado e tempo de uso (experiência) do Facebook. Destaca-se que a "idade", o "gênero" e a "experiência de uso" são moderadores e foram considerados a partir do cálculo de média das respostas, ou seja, dividindo-se as respostas em dois grupos com base na média geral de idades e o valor médio de utilização diária do Facebook.

Os dados foram tabulados e analisados estatisticamente por meio do softwareStatistical Package for the Social Sciences (SPSS), versão 21.0. A análise de resultados foi realizada sob duas perspectivas para atingir aos objetivos propostos neste estudo. Na primeira, a fim de demonstrar a percepção dos usuários em relação ao uso do Facebook, foram analisadas as estatísticas descritivas das variáreis e fatores propostos por Visentini et al. (2016). Na segunda, buscou-se verificar a influência dos moderadores (idade, gênero e experiência de uso) na prática de uso do Facebook. Para a realização das análises, aplicou-se testes $\mathrm{t}$ de amostras independentes, que avalia se as diferenças observadas entre as médias de duas amostras ocorrem por acaso ou se houve uma diferença verdadeira (HAIR Jr. et al., 2009).

\section{Análise e Discussão dos Resultados}

\subsection{Análise descritiva da percepção dos usuários}

A fim de atingir o objetivo geral proposto, foram realizadas análises descritivas.Demonstra-se que esse tipo de análise de dados possibilitará a caracterização da percepção dos usuários em relação a aspectos do Facebook. Foram considerados as médias e os desvios-padrão das variáveis e dos fatores,os quais são apresentados na tabela 1. Além disso, optou-se por considerar os valores do alphade Cronbach de cada fator, a fim de demonstrar sua aplicabilidade e confiabilidade no estudo, uma vez que esse índice é uma medida da confiabilidade da consistência interna do conjunto de itens, e valores acima de 0,6 são considerados satisfatórios (MALHOTRA, 2012). 
Tabela 1 - Médias, desvios-padrão e alpha de Cronbach das variáveis e fatores analisados

\begin{tabular}{|c|c|c|c|}
\hline Constructos & Variáveis & Média & $\begin{array}{l}\text { Desvio- } \\
\text { Padrão }\end{array}$ \\
\hline \multirow{5}{*}{$\begin{array}{l}\text { Facilidade de uso } \\
\text { alpha de Cronbach } \\
\text { do fator }=0,810\end{array}$} & Aprender a usar o Facebook é fácil. & 6,16 & 0,984 \\
\hline & $\begin{array}{l}\text { O Facebook é uma ferramenta compreensível e clara de se } \\
\text { usar. }\end{array}$ & 5,95 & 0,955 \\
\hline & É fácil se tornar habilidoso no uso do Facebook. & 5,97 & 0,925 \\
\hline & É fácil fazer o Facebook executar o que eu quero. & 5,64 & 1,090 \\
\hline & Média geral para o fator & 5,93 & 0,790 \\
\hline \multirow{5}{*}{$\begin{array}{l}\text { Utilidade no } \\
\text { trabalho } \\
\text { alphade } \\
\text { Cronbachdo fator }= \\
0,746\end{array}$} & $\begin{array}{l}\text { Usar o Facebook me permite terminar tarefas mais } \\
\text { rapidamente. }\end{array}$ & 3,81 & 1,744 \\
\hline & Usar o Facebook aumenta a minha produtividade. & 3,04 & 1,544 \\
\hline & $\begin{array}{l}\text { 9. Usar o Facebook é útil para o meu trabalho/ atividades } \\
\text { acadêmicas. }\end{array}$ & 4,71 & 1,538 \\
\hline & 10. Em geral, o Facebook é útil. & 5,24 & 1,276 \\
\hline & Média geral para o fator & 4,20 & 1,156 \\
\hline \multirow{3}{*}{$\begin{array}{l}\text { Utilidade } \\
\text { informacional } \\
\text { alphade Cronbach } \\
\text { do fator }=0,740\end{array}$} & $\begin{array}{l}\text { Usar o Facebook me permite adquirir mais informações ou } \\
\text { conhecer outras pessoas. }\end{array}$ & 5,70 & 1,195 \\
\hline & $\begin{array}{l}\text { 8. Usar o Facebook aumenta a minha eficiência em } \\
\text { compartilhar informações. }\end{array}$ & 5,88 & 1,130 \\
\hline & Média geral para o fator & 5,79 & 1,035 \\
\hline \multirow{4}{*}{$\begin{array}{l}\text { Entretenimento } \\
\text { alphade } \\
\text { Cronbachdo fator }= \\
0,836\end{array}$} & 11. Usar o Facebook me proporciona muita diversão. & 4,75 & 1,283 \\
\hline & 12. Eu acho o Facebook muito divertido. & 4,41 & 1,380 \\
\hline & 13. O Facebook é entusiasmante. & 4,06 & 1,282 \\
\hline & Média geral para o fator & 4,40 & 1,142 \\
\hline \multirow{4}{*}{$\begin{array}{l}\text { Imersão focada } \\
\text { alphadeCronbachdo } \\
\text { fator }=0,658\end{array}$} & $\begin{array}{l}\text { 14. Eu fico tão distraído no Facebook que eu esqueço de todo } \\
\text { o resto. }\end{array}$ & 3,43 & 1,675 \\
\hline & $\begin{array}{l}\text { 15. Quando estou no Facebook, fico focado(a) na atividade } \\
\text { que estou fazendo no Facebook. }\end{array}$ & 4,06 & 1,499 \\
\hline & $\begin{array}{l}\text { 16. Quando estou no Facebook, minha atenção não é } \\
\text { facilmente desviada. }\end{array}$ & 3,62 & 1,553 \\
\hline & Média geral para o fator & 3,70 & 1,215 \\
\hline \multirow{6}{*}{$\begin{array}{l}\text { Intenção } \\
\text { comportamentalde } \\
\text { uso } \\
\text { alphade } \\
\text { Cronbachdo fator = } \\
0,828\end{array}$} & $\begin{array}{l}\text { 17. Eu pretendo continuar usando o Facebook no futuro } \\
\text { próximo. }\end{array}$ & 5,44 & 1,242 \\
\hline & 18. Eu planejo usar o Facebook no futuro distante. & 4,67 & 1,514 \\
\hline & $\begin{array}{l}\text { 19. Eu pretendo recomendar o Facebook para os meus } \\
\text { amigos no futuro. }\end{array}$ & 4,27 & 1,513 \\
\hline & 20. Acredito que irei usar o Facebook nos próximos meses. & 5,98 & 1,047 \\
\hline & 21. Eu espero que o meu uso do Facebook continue no futuro. & 5,05 & 1,467 \\
\hline & Média geral para o fator & 5,08 & 1,053 \\
\hline
\end{tabular}

Fonte: Elaborado pelos autores com base em Visentini et al. (2016).

A partir dos resultados da tabela 1, destacam-se as maiores e menores médias, tanto para os fatores quanto para as variáveis, com o intuito de explorar tais achados e relacioná-los com a literatura pertinente.

Considerando a perspectiva de que a velocidade, a funcionalidade, a praticidade e o imediatismo pautam as pequenas escolhas dos usuários em sua navegação cotidiana pelo ciberespaço (FUSER; PERSINA Jr., 2009), evidencia-se que o Facebook é uma ferramenta fácil de utilizar, constatação indicada pela média elevada $(5,93)$ e pelo baixo desvio-padrão $(0,984)$ do fator "facilidade de uso". O desvio-padrão determina o quanto variam as respostas dos entrevistados em relação à média e, se o valor obtido for 
menor que 1, indica que os respondentes tendem a ter opiniões semelhantes sobre o mesmo assunto.Se ele for maior que 1, significa que os respondentes divergem entre si (HAIR Jr. et al., 2009).

Em contraste, o fator "imersão focada"obteve a menor média $(3,70)$ e o maior desviopadrão $(1,215)$, o que implica que os respondentes apresentam maior divergência nas respostas e não percebem expressivamente que o Facebook é uma forma de imersão, ou seja, durante o acesso e a navegação nessa rede social, o usuário permanece atento aos assuntos externos, que não fazem parte da ferramenta.

Entre as variáveis de maior média (variável 1 - média de 6,16; 20 -5,98), a primeira obteve a média mais elevada,demonstrando que a rede social apresenta facilidade de utilização, além de diversos recursos acessíveis por meio de um navegador web, sem que haja necessidade de instalação de software adicional (NASCIMENTO JÚNIOR; PIMENTEL; DOTTA; BRAGA, 2013). A variável 20 obteve a segunda maior média, o que fortalece a premissa de que o Facebook é percebido pelos brasileiros como um mecanismo para compartilhar dados e informações, além de constituir um ambiente no qual são estabelecidos e mantidos relacionamentos (OLIVEIRA, HUERTAS, 2014), motivando, assim, o retorno dos usuários para a rede.

Entre as variáveis de menor média (variável 6 - média de 3,04; 14 -3,43), a sexta variável, integrante do fator "utilidade no trabalho", obteve a menor média. Analisando a maioria das médias das variáveis dos fatores "utilidade informacional" e "utilidade no trabalho", constata-se que, de forma geral, a utilidade da rede social é valorizada pelos usuários, mas a plataforma contribui pouco para o aumento de sua produtividade. Considerando o ambiente organizacional, Barbosa (2014) afirma que existem pessoas que não conseguem estabelecer um limite para o acesso ao Facebook durante o horário de expediente e, assim, prejudicam sua produtividade devido ao tempo destinado à rede social. A variável 14 apresentou a segunda menor média, indicando que os acadêmicos não se sentem imersos na rede social. Uma explicação possível para esse achado é dada por Bitencourt (2012) ao considerar os nativos digitais, aqueles que cresceram com as tecnologias digitais presentes em sua vivência, os quais desenvolvem atividades simultaneamente, sendo capazes de atuar com atenções múltiplas, o que não necessitaria de imersão profunda na rede social para a realização das tarefas.

Ademais, a confiabilidade de cada um dos fatores foi verificada utilizando-se do alphade Cronbach. Destaca-se que valores superiores a 0,658 foram obtidos em todos os fatores, o que demonstra que o valor do alpha pode ser considerado bom para os constructos "utilidade no trabalho" e "utilidade informacional" (superiores a 0,740 ) e muito bom para os fatores "facilidade de uso", "entretenimento" e "intenção comportamental de uso"(superiores a 0,810), conforme recomendação de Hair Jr. et al. (2009). Apenas o fator "imersão focada" obteve valor do alpha abaixo de 0,70. Destaca-se que os alphas apresentaram valores similares aos do estudo de Visentini et al. (2016), corroborando a confiabilidade dos constructos teóricos, assim como a consistência interna do conjunto de itens analisados.

Além de identificar a percepção dos usuários do Facebook em relação a aspectos relevantes da literatura, torna-se instigante verificar diferenças nas suas percepções considerando características como gênero, idade e experiência de uso. A partir da evidência de proposições a respeito de um grupo de indivíduos, neste caso,usuários de determinada rede social com características comuns, é possível estabelecer estratégias de comunicação direcionadas a esse público. Dessa forma, a próxima seção dedicase a identificação de influências dos moderadores "gênero", "idade" e "experiência no uso" do Facebook.

\subsection{Gênero, idade e experiência como moderadores do uso do facebook}

Tendo em vista a composição dos fatores e variáveis, as médias atribuídas pelos respondentes foram comparadas por meio do teste $t$ de amostras independentes, com o intuito de identificar diferenças significativas associadas aos moderadores "gênero", "idade" e "experiência de uso" com o Facebook. Considerando, inicialmente, os testes com o moderador "gênero", apresenta-se na tabela 2 as médias e os desvios-padrão para homens e mulheres das variáveis significativas (sig. $\leq 0,05)$ para o teste $t$. 
Tabela 2 - Testes t para diferenças de percepção em relação ao gênero

\begin{tabular}{l|l|c|c|c|c|c|c}
\hline $\begin{array}{l}\text { Fator a que } \\
\text { pertencem } \\
\text { as variáveis }\end{array}$ & Variável & \multicolumn{2}{|c|}{ Homens } & \multicolumn{2}{c|}{ Mulheres } & \multicolumn{3}{c}{ Teste $t$} \\
\hline & & Média & $\sigma$ & Média & $\sigma$ & Valor & Sig. \\
\hline & 1. Aprender a usar o Facebook é fácil. & 6,00 & 1,106 & 6,26 & 0,889 & $-2,781$ & 0,006 \\
\cline { 2 - 9 } & $\begin{array}{l}\text { 2. O Facebook é uma ferramenta } \\
\text { compreensível e clara de se usar. }\end{array}$ & 5,78 & 1,007 & 6,06 & 0,906 & $-3,118$ & 0,002 \\
\cline { 2 - 9 } & $\begin{array}{l}\text { F. É fácil se tornar habilidoso no uso do } \\
\text { uso }\end{array}$ & 5,85 & 0,977 & 6,04 & 0,886 & $-2,071$ & 0,044 \\
\hline $\begin{array}{l}\text { Imersão } \\
\text { focada }\end{array}$ & $\begin{array}{l}\text { 14. Eu fico tão distraído no Facebook } \\
\text { que eu esqueço de todo o resto. }\end{array}$ & 3,16 & 1,646 & 3,59 & 1,674 & $-2,648$ & 0,008 \\
\hline
\end{tabular}

Fonte: Elaborado pelos autores com base nos dados da pesquisa.

Observa-se que quatro questões apresentaram diferenças significativas, sendo três pertencentes ao fator "facilidade de uso" e uma ao fator "imersão focada". As mulheres atribuíram maior média (média 6,26; sig. 0,006) para a variável 1, demonstrando que o gênero feminino tende a considerar mais fácil aprender a usar o Facebook em comparação ao gênero masculino. Corroborando, Venkatesh e Morris (2000) identificaram que, para as mulheres, o fator dominante no uso de softwares é a "facilidade de uso percebida", o que indica que esse aspecto é valorizado pelas mulheres e tende a motivar a utilização do Facebook, uma vez que é considerado fácil aprender seu uso.

Maior média também foi obtida pelo gênero feminino nas variáveis 2 e 3,com valores de 6,06 (sig. 0,002) e 6,04 (sig. 0,044) respectivamente.Kaufmann (2005) contribui para tal achado ao afirmar que as mulheres possuem mais condições facilitadas para o uso da tecnologia da informação em comparação aos homens.

Adicionalmente, as mulheres atribuíram maior média (média 3,59; sig. 0,008) para a variável 14, em comparação aos homens, embora o valor alcançado esteja próximo da metade da escala, representando que a distinção do gênero feminino é discreta. Fortalecendo esse resultado, Bandeira e Garcez (2013) verificaram que mulheres compreendidas na faixa etária de 18 a 25 anos compartilham mais publicações com conteúdo de humor para se divertir ou divertir os amigos.

Na sequência, são consideradas as diferenças de percepção entre respondentes de diferentes idades. Na tabela 3, estão apresentadas as variáveis que diferiram significativamente (sig. $\leq 0,05)$, com suas respectivas médias, desvios-padrão, teste t e significância.

Nota-se que os usuários mais novos, aqueles que possuem 20 anos ou menos, atribuíram maior concordância na variável 3 (média 6,05; sig. 0,029) em comparação aos usuários de 21 anos ou mais. $\mathrm{Na}$ concepção de Tapscott (1999), os jovens demonstram curiosidade diante das novidades tecnológicas e apresentam facilidade em utilizar diferentes recursos. Corroborando, Grohmann, Silinske, Marquetto e Battistella (2015) verificaram que, para os jovens, a "facilidade de uso percebida" em relação ao iPad exerce relevante influência para sua intenção de utilizá-lo. 
Tabela 3 - Testes t para diferenças de percepção em relação à idade

\begin{tabular}{l|l|l|l|l|l|l|l}
\hline \multirow{2}{*}{$\begin{array}{l}\text { Fator a que } \\
\text { pertencem as } \\
\text { variáveis }\end{array}$} & \multirow{2}{*}{ Variável } & \multicolumn{2}{l|}{ Até 20 anos } & \multicolumn{2}{l}{ Acima de 21 } & \multicolumn{2}{l}{ Teste $t$} \\
\cline { 3 - 9 } & & Média & $\sigma$ & Média & $\sigma$ & Valor & Sig. \\
\hline \multirow{2}{*}{ Facilidade de uso } & $\begin{array}{l}\text { 3. É fácil se tornar habilidoso no } \\
\text { uso do Facebook. }\end{array}$ & 6,05 & 0,868 & 5,86 & 0,990 & 2,185 & 0,029 \\
\cline { 2 - 9 } & $\begin{array}{l}\text { 4. É fácil fazer o Facebook } \\
\text { executar o que eu quero. }\end{array}$ & 5,78 & 1,009 & 5,46 & 1,170 & 3,171 & 0,002 \\
\hline Utilidade no trabalho & $\begin{array}{l}\text { 5. Usar o Facebook me permite } \\
\text { terminar tarefas mais rapidamente. }\end{array}$ & 6,23 & 0,915 & 6,03 & 0,989 & 2,200 & 0,028 \\
\hline
\end{tabular}

Fonte: Elaborado pelos autores com base nos dados da pesquisa

Ratificando a premissa que os mais jovens apresentam maior percepção de "facilidade de uso" em relação ao Facebook, verificou-se que eles alcançaram maior média (média 5,78; sig. 0,002) na variável 4. Dias (2000) fortalece tal achado ao afirmar que equipamentos de tecnologia, como os computadores, necessitam apresentar facilidade de uso ao passo que permitem a ampliação da qualidade do trabalho, agilidade na execução de tarefas e melhora da produtividade.

Adicionalmente, foi demonstrado que os usuários mais novos utilizam a rede social considerada para fins laborais e acadêmicos, conforme resultado da variável 5 (média 6,23; sig. 0,028). Evidencia-se, assim, o caráter contributivo do Facebook para um melhor desempenho acadêmico e profissional dos discentes ao permitir acesso instantâneo a uma variedade de informações, assim como localizar perfis de pessoas.

A exposição e a experiência de uso de determinada tecnologia pode tornar o usuário mais habilidoso, assim como propiciar alterações na sua percepção em relação ao meio de comunicação utilizado (KARAHANNA; STRAUB; CHERVANY, 1999). Partindo dessa concepção, buscou-se identificar as diferenças de percepção dos respondentes considerando sua experiência de uso do Facebook. Para tanto, a experiência de uso foi associada ao tempo diário de utilização dessa rede social virtual.A partir do valor médio de utilização diária do Facebook, verificaram-se diferenças de percepção dos usuários, e as variáveis que diferiram significativamente (sig. $\leq 0,05)$ estão apresentadas na tabela 4 com suas respectivas médias, desvios-padrão, teste t e significância.

Tabela 4 - Testes t para diferenças de percepção em relação à experiência de uso

\begin{tabular}{l|l|l|l|l|l|l|l|l}
\hline $\begin{array}{l}\text { Fator a que } \\
\text { pertencem as } \\
\text { variáveis }\end{array}$ & \multirow{2}{*}{ Variável } & \multicolumn{2}{l|}{ Até 2,44 horas } & \multicolumn{2}{l|}{ A partir 2,45 } & \multicolumn{2}{l}{ Teste $t$} \\
\cline { 3 - 9 } & & Média & $\sigma$ & Média & $\sigma$ & Valor & Sig. \\
\hline $\begin{array}{l}\text { Facilidade de } \\
\text { Uso }\end{array}$ & $\begin{array}{l}\text { 2. O Facebook é uma ferramenta } \\
\text { compreensível e clara de se usar. }\end{array}$ & 5,87 & 0,991 & 6,07 & 0,902 & $-2,248$ & 0,023 \\
\hline $\begin{array}{l}\text { Utilidade } \\
\text { Informacional }\end{array}$ & $\begin{array}{l}\text { 8. Usar o Facebook aumenta a } \\
\text { minha eficiência em compartilhar } \\
\text { informações. }\end{array}$ & 5,75 & 1,170 & 6,05 & 1,060 & $-2,817$ & 0,005 \\
\hline Entretenimento & 13. O Facebook é entusiasmante. & 4,59 & 1,328 & 4,94 & 1,209 & $-3,033$ & 0,004 \\
\hline Imersão Focada & $\begin{array}{l}\text { 14. Eu fico tão distraído no } \\
\text { Facebook que eu esqueço de todo } \\
\text { o resto. }\end{array}$ & 3,17 & 1,433 & 3,77 & 1,275 & $-3,856$ & 0,000 \\
\hline
\end{tabular}




\begin{tabular}{|c|c|c|c|c|c|c|c|}
\hline \multirow{2}{*}{$\begin{array}{l}\text { Fator a que } \\
\text { pertencem as } \\
\text { variáveis }\end{array}$} & \multirow{2}{*}{$\begin{array}{l}\text { Variável } \\
\text { 17. Eu pretendo continuar usando o } \\
\text { Facebook no futuro próximo. }\end{array}$} & \multicolumn{2}{|c|}{ Até 2,44 horas } & \multicolumn{2}{|c|}{ A partir 2,45} & \multicolumn{2}{|l|}{ Teste $t$} \\
\hline & & 5,29 & 1,317 & 5,63 & 1,118 & $-2,928$ & 0,003 \\
\hline \multirow{4}{*}{$\begin{array}{l}\text { Intenção } \\
\text { Comportamental } \\
\text { de Uso }\end{array}$} & $\begin{array}{l}\text { 18. Eu planejo usar o Facebook no } \\
\text { futuro distante. }\end{array}$ & 4,45 & 1,577 & 4,92 & 1,389 & $-3,324$ & 0,001 \\
\hline & $\begin{array}{l}\text { 19. Eu pretendo recomendar o } \\
\text { Facebook para os meus amigos no } \\
\text { futuro. }\end{array}$ & 4,13 & 1,543 & 4,42 & 1,453 & $-2,042$ & 0,042 \\
\hline & $\begin{array}{l}\text { 20. Acredito que irei usar o } \\
\text { Facebook nos próximos meses. }\end{array}$ & 5,87 & 1,098 & 6,13 & 0,966 & $-2,647$ & 0,008 \\
\hline & $\begin{array}{l}\text { 21. Eu espero que o meu uso do } \\
\text { Facebook continue no futuro. }\end{array}$ & 4,87 & 1,552 & 5,27 & 1,324 & $-2,913$ & 0,004 \\
\hline
\end{tabular}

Fonte: Elaborado pelos autores com base nos dados da pesquisa

Destaca-se que os usuários mais experientes, aqueles que utilizam a rede social 2,45 horas ou mais diariamente, atribuíram maior média (média 6,07; sig. 0,023) na variável 2. Li e Lindner (2007) fortalecem esse resultado ao afirmarem que as experiências prévias com o uso de tecnologias da informação e comunicação (TICs) contribuem para o sucesso dessas iniciativas, uma vez que os usuários precisam se confortáveis e perceber novas possibilidades de utilização que lhes sejam familiares.

Os acadêmicos que acessam por mais tempo o Facebook demonstram maior concordância com a variável 8 (média 6,05; sig. 0,005), tendo em vista que é considerável o papel da experiência na construção de conhecimentos e competências. Conforme Dewey (1979), a experiência é fundamental na construção da cognição, uma vez que existe conexão orgânica entre educação e experiência, suportada pelo conhecimento relevante que modifica a perspectiva, a atitude e a competência do indivíduo. Dessa forma, apoia-se a premissa de que os usuários do Facebook desenvolvem competências, como o compartilhamento de informações, a partir da experiência que possuem com a plataforma digital.

Complementarmente, a variável 13, pertencente ao fator "entretenimento", teve a média mais elevada (média 4,94; sig. 0,004) pelos usuários mais experientes. Neste sentido, Zago e Bastos (2013) verificaram, ao compararem notícias mais repercutidas no Twitter e no Facebook em jornais do Brasil, Alemanha, Espanha, Estados Unidos e Reino Unido, que essa última rede social é utilizada majoritariamente para conteúdos ligados ao entretenimento e curiosidades, enquanto o Twitter é majoritariamente empregado para cobertura de eventos políticos, econômicos e sociais. Ademais, Abedniya e Mahmouei (2010) defendem que os utilizadores das redes sociais percebem sua tendência em proporcionar sentimento de divertimento e de prazer, fomentando sua intenção de continuidade de uso.

Os usuários mais experientes também atribuíram maior média (média 3,77; sig. 0,000) para a variável 14. Conforme se observa na tabela 4 , no entanto, as médias da variável permaneceram próximas do valor da metade da escala, evidenciando-se que os respondentes se mostram indiferentes em relação à afirmação. A diferença significativa verificada demonstra uma tendência dos indivíduos que a utilizam por mais tempo diariamente de perceberem que é possível distrair-se e esquecer-se de aspectos inerentes as suas vidas.

Observa-se que as cinco afirmações $(17,18,19,20$ e 21) do fator "intenção comportamental de uso" apresentaram diferenças significativas(sig. $\leq 0,05$ ), sendo que os usuários que utilizam o Facebook diariamente por mais tempo obtiveram maiores médias (médias de 5,63; 4,92; 4,42; 6,13; 5,27; sig. 0,$003 ; 0,001 ; 0,042 ; 0,008 ; 0,004$, respectivamente).Isso demonstra que, quanto mais a pessoa se torna experiente, maior é sua intenção de continuar acessando a rede social. Corroborando, Kaufmann (2005) identificou que, quanto maior a experiência do usuário em relação à determinada tecnologia da informação, maior é o uso que faz da mesma. 
Por fim, destaca-se que Almansa, Fonseca e Castillo (2013) fortalecem o resultado da variável 19ao constatarem que o Facebook possibilita o relacionamento de jovens com seus círculos de amizade mais próximos, o que indica que os usuários selecionam seus amigos próximos para se comunicarem virtualmente.Dessa forma, a experiência de uso foi responsável pelo impacto na percepção dos acadêmicos em nove variáveis, indicando ser um moderador relevante na compreensão do comportamento do usuário de redes sociais virtuais.

Os moderadores considerados propiciaram diferenças significativas (sig. $\leq 0,05)$ nas percepções dos usuários do Facebook, especialmente, em relação às variáveis compreendidas nos fatores "Facilidade de Uso" e "Intenção Comportamental de Uso". Ressalta-se que as médias foram elevadas em um dos grupos de moderadores.No caso do gênero, as mulheres atribuíram maior média às variáveis que diferiram significativamente; enquanto que, para a idade, os indivíduos mais jovens foram responsáveis pelas maiores médias; $\mathrm{e}$, considerando a experiência de uso, os usuários mais experientes demonstraram maior concordância com as variáveis. Demonstram-se, assim, três segmentos de mercado que são potenciais para a utilização do Facebook como ferramenta de comunicação.

\section{Conclusão}

Esta pesquisa visou identificar a percepção de usuários brasileiros com relação aos motivadores do uso do Facebook, os quais foram apresentados primeiramente por Visentini et al. (2016), bem como analisar de que modo os moderadores idade, gênero e experiência de uso na rede social influenciam essa percepção. Entende-se que os achados deste estudo podem contribuir na melhor compreensão, por parte de gestores e de organizações, de como realizar a aproximação dos clientes mediante o uso de plataformas virtuais de relacionamento. Além disso, para o campo da investigação em Marketing, Sistemas de Informação e Administração da Informação, apresentam-se achados atuais e a direcionados ao contexto nacional para subsidiar o desenvolvimento de novos estudos.

A condução do estudo foi realizada por meio de um levantamento, aplicado aos acadêmicos de duas instituições federais de ensino superior do estado do Rio Grande do Sul. A amostra não probabilística de usuários da rede social Facebook foi composta por 454 respondentes. Na análise de resultados, para atingir os objetivos propostos, primeiramente analisou-se a percepção dos respondentes no que se refere às variáveis e aos fatores propostos por Visentini et al. (2016)por meio da estatística descritiva. Na sequência, verificou-se a influência do gênero, da idade e da experiência de uso na prática de uso do Facebook mediante a aplicação da estatística paramétrica.

Os resultados da primeira etapa da pesquisa indicaram que esta rede social possui uma plataforma de fácil uso, mas que não incita imersão por parte de seus usuários. Ademais, embora os jovens apresentem boas relações com o ambiente descomplicado da rede social para o desenvolvimento de suas atividades, ela não contribui significativamente para a produtividade de seus usuários.

$\mathrm{Na}$ segunda etapa da pesquisa, referente aos moderadores, observou-se que o gênero feminino apresenta maior intenção de uso da rede social quando se mostra de fácil uso, e que, além disso, as mulheres possuem distinção em relação aos homens quanto à imersão com a rede social, de modo que o gênero feminino está associado com maior pujança aos fatores "facilidade de uso" e "imersão focada". Com relação ao moderador "idade", destaca-se a associação entre jovens e sua facilidade em aprender e utilizar a rede social Facebook. Ademais, os usuários com 20 anos ou menos apresentam maior uso da rede de relacionamentos para desempenhar suas atividades acadêmicas e laborais.

Por fim, ao verificar a associação das variáveis com o moderador "experiência", percebeu-se que os usuários que passam mais tempo utilizando a rede social tornam-se mais habilidosos em seu uso, e que também incute em sua capacidade de desenvolver conteúdo e externá-los na rede social. Os resultados também implicam que, quanto mais tempo de uso é dispensado diariamente à rede social, mais os usuários estão dispostos a utilizá-la. De modo geral, a partir dos resultados obtidos, este estudo contribui com a compreensão acerca dos fatores que motivam a intenção comportamental de uso do Facebook no Brasil considerando diferentes características dos seus usuários, neste caso, a idade, o gênero e a experiência de 
uso. Tais resultados podem fornecer subsídios para que as empresas formulem estratégias mercadológicas digitais adequadas a cada tipo de público. As redes sociais virtuais revelam ser um mecanismo poderoso na captação e manutenção dos clientes, entendendo-se que o direcionamento de postagens e ofertas a partir desses mecanismos tende a gerar resultados positivos às organizações.

Alguns atores limitaram a generalização dos resultados deste estudo, como a investigação apenas da plataforma digital Facebook, não considerando outras redes sociais. Entende-se que é necessária a ampliação do objeto de análise considerando outras plataformas virtuais e os possíveis fatores que podem justificar o seu uso. Além disso, o estudo apresentou limitação quanto à amostra, homogênea, composta apenas por estudantes de ensino superior.

Estas limitações não desqualificam esta pesquisa, mas indicam que há melhorias a serem realizadas na compreensão dos motivadores do uso do Facebook, bem como dos moderadores deste uso.Nesse sentido, indicam a necessidade de estudos futuro a fim de expandir a investigação. Assim, sugere-se ampliar a investigação para a compreensão de outras redes sociais, ou outras mídias digitais, como aplicativos de celular. Além disso, a investigação de outros públicos também se faz necessária para expandir as considerações da pesquisa. Por fim, sugere-se que outros moderadores de uso de tecnologias sejam mensurados, ampliando a perspectiva proposta nesta investigação.

\section{Referências}

ABEDNIYA, A.; MAHMOUEI, S. The impact of social networking websites to facilitate the effectiveness of viral marketing. International Journal of Advanced Computer Science and Applications, [S.I], v. 1, n. 6, p. 139-146, 2010.

AGARWAL, R.; KARAHANNA, E. Time Flies When You're Having Fun: Cognitive Absorption and Beliefs about Information Technology Usage. MIS Quarterly, [S.I], v. 24, n.4, p. 665-694, 2000.

ALMANSA, A.; FONSECA, O.; CASTILLO, A. Redes sociales y jóvenes. Uso do Facebook em la juventud colombiana y española. Revista Científica Educomunicácion, [S.I], v. 20, n. 40, p. 127-135, 2013.

BAKER, M. J. Selecting a Research Methodology. The Marketing Review, [S.I], v. 1, n. 3, p. 373-397, 2001.

BANDEIRA, M. O. R.; GARCEZ, R. O. Como comunicar o cliente através do Facebook: O caso da fanpage da Trifil.In: CONGRESSO DE CIÊNCIAS DA COMUNICAÇÃO NA REGIÃO SUL, 14., 2013, Santa Cruz do Sul. Anais... Santa Cruz do Sul: Intercom, 2013.

BARBOSA, C. Saiba Estipular um Limite para as Redes Sociais no Trabalho. 2014.

Disponível em: <http://www.administradores.com.br/artigos/carreira/saiba-estipular-um-limite-para-asredes-sociais-no-trabalho/75789/>. Acesso em: 29 jun. 2016.

BOLAR, K. P. Motives behind the use of social networking sites: an empirical study. The IUP Journal of Management Research, [S.I], v. 8, n. 1, p. 75-84, 2009.

BITENCOURT, R. B. Novas tecnologias, novas educações. Revista Contexto Educação, [S.I], v. 3, n. 4, p. 110-112, 2012.

CAN, L.; KAYA, N.; Social networking sites addiction and the effect of attitude towards social network advertising. Procedia-Social and Behavioral Sciences, [S.I],v. 235, n. 1, p. 484-492, 2016.

COMSCORE. (2014). Brazil Digital Future in Focus 2014.2014. Disponível em: < http://www.comscore. com/por/Imprensa-e-eventos/Apresentacoes-e-documentos/2014/2014-Brazil-Digital-Future-in-FocusWebinar >. Acesso em: 2 maio 2015.

CORRAR, L. J.; PAULO, E.; DIAS FILHO, J. M. Análise multivariada: para os cursos de administração, ciências contábeis e economia. São Paulo: Atlas, 2007. 
CORREA, R. S.; PINTO, M. R.; BATINGA. G. L. A Beleza na escuridão: um "olhar" sobre a experiência de consumo por mulheres deficientes visuais em serviços de beleza e estética.

Revista Ciências Administrativas, [S.I], v. 22, n. 2, p. 371-395, 2016.

CORREA, T.; HINSLEY, A. W.; ZUNIGA, H. G. Who interacts on the Web? The intersection of users'personality and social media use. Computers in Human Behavior, [S.I], v. 26, n. 2, 2010.

DEWEY, J. Como pensamos: como se relaciona o pensamento reflexivo com o processo educativo: uma reexposição. 4. ed. São Paulo: Nacional, 1979.

DIAS, D. S. Motivação e resistência ao uso da tecnologia da informação: um estudo entre gerentes. RAC - Revista Administração Contemporânea, [S.I], v. 4, n. 2, p. 51-66, 2000.

ELIAS, S. M.; SMITH, W. L.; BARNEY, C. E. Age as a moderator of attitude towards technology in the workplace: work motivation and overall job satisfaction. Behaviour and Information Technology, [S.I], v. 31, n. 5, p. 453-467, 2012.

FALAHAH, D. R. Study of Social Networking usage in Higher Education Environment. Procedia- Social and Behavioral Siences, [S.I],v. 67, n. 10, p. 156-166, 2012.

FALAHAHA; ROSMALA, D. Study os Social Networking Usage in Higher Education Environment.

Procedia- Social and Behavioral Scineces, [S.I],v. 67, p. 156-166, 2012.

FIELD, A. Descobrindo a estatística usando SPSS. 2. ed. Porto Alegre: Artmed, 2009.

FUSER, B.; PERSINA, C. Comunicação e tecnologias. Rio de Janeiro: E - papers, 2009.

GALLUCH, P. S.; TATCHER, J. B. Slacking and the Internet in the Classroom: A

Preliminary Investigation. In: WORKSHOP ON HCI RESEARCH IN MIS, 5., 2006, Milwaukee.

Proceedings... Milwaukee: Winsconsin, 2006, p. 25-29.

GROHMANN, M. Z. et al Prazer, beleza, marca, facilidade, utilidade... o que importa para os jovens em um iPad? REMark -Revista Brasileira de Marketing, [S.I], v. 14, n. 4, p. 451 - 467, 2015.

GUJARATI, D. N. Econometria básica. 3. ed. São Paulo: Makron Books, 2000.

HAIR Jr., F. et al. Fundamentos de Métodos de Pesquisa em Administração. Porto Alegre: Bookman, 2009.

HART, J.; SUTCLIFFE, A. G.; ANGELI, A. Love it or hate it! Interactivity and user types. In:SIGCHI CONFERENCE ON HUMAN FACTORS IN COMPUTING SYSTEMS, 13., 2013, New York.

Proceedings... New York: ACM, 2013. p. 2059-2068.

JI, Y.;WANG, G.;ZHANG, Q.;ZHU,Z. Online social networking behaviors among Chinese younger and older adolescent: the influence of age, gender, personality, and attachment styles. Computers in Human Behavior, [S.I],v. 41, n. 1, p. 393-402, Dec. 2014.

JOINER, R., et al. Publically different, privately the same: gender differences and similarities in response to Facebook status updates. Computers in Human Behavior, [S.I], v. 39, n.1, p. 165-169, 2014.

KARAHANNA, E.; STRAUB, D. W.; CHERVANY, N.L. Information Technology Adoption Across Time: A cross-sectional comparison of pre-adoption and post-adoption beliefs.Mis Quarterly, [S.I], v. 23, n. 2, p.183-213, jun. 1999.

KAUFMANN, S. M. A. Tecnologia da informação em uma instituição de ensino superior: fatores que influenciam sua utilização. 117 f. 2005. Dissertação (Mestrado em Administração) - Universidade Federal do Rio Grande do Sul, Porto Alegre, 2005.

KAVOURA, A.; STAVRIANEA, A. Following and Belonging to an Online Travel ommunity in Social Media, its Shared Characteristics and Gender Differences. Procedia- Social and Behavioral Sciences, [S.I], v. 175, n. 1 , p. $515-521,2015$. 
LABIB, N. M.; MOSTAFA, R. H. A. Determinants of Social Networks Usage in Collaborative Learning: Evidence from Egypt. Procedia Computer Science, [S.I], v. 65, n. 1, p. 432-441, 2015.

LEVINE, D. M.; BERENSON, M. L.; STEPHAN, D. Estatística: teoria e aplicações. Rio de Janeiro: LTC, 2000.

LI, Y.; LINDNER, J. R. Faculty adoptio behavior about webbased distance education: a case study from China Agricultural University. British Journal of Educational Technology, [S.I], v. 38, n. 1, p. 83 - 94 , 2007.

LIAO, C. H. Exploring the impact of age and usage experience of e-service on user perceived web quality. Lecture Notes in Computer Science, [S.I], v. 9193, n. 1, p.230-238. 2015.

LIN, K.; LU, H. Why people use social networking sites: An empirical study integrating network externalities and motivation theory, Computers in Human Behavior, [S.I], v. 27, n. 3, p. 1152-1161, 2011.

LÖBLER, M.L.; VISENTINI, M. S.; ESTIVALETE, V. F. B. A vision of Orkut's users: studying this phenomenon through cognitive absorption. Journal of Information Systems and Technology Management, [S.I], v. 8, n.1, art. 3, p. 51-72, 2011.

MALHOTRA, N. K. Pesquisa de marketing: uma orientação aplicada. 3. ed. Porto Alegre: Bookman, 2006.

MARUPING, L.M., MAGNI, M. What's the Wheather like? The effect of team learning climate, Empowerment Climate, and Gender on Individual's Technology Exploration and Use. Journal of Management Information Systems, [S.I], v. 29, n. 1, 2012.

MORAES, G.H.S.M.; CAPPELLOZZA, A.; MEIRELLES, F.S. \#VEMPRARUA- A Tecnologia da informação e as manifestações sociais: um estudo da utilização das redes sociais para participação dos protestos. In: ENCONTRO NACIONAL DA ANPAD, 38., 2014, Rio de Janeiro. Anais... Rio de Janeiro: EnANPAD, 2014. p.1-16.

MORRIS, M.G.; VEKATESH, V. Age differences in technology adoption decisions: implications for a changing work force. Personnel Psychology, [S.I], v. 53, n. 1, 2000.

MORRIS, M.G.; VENKATESH, V.; ACHERMAN, P.L. Gender and age differences in employee decisions about new technology: an extension to the theory of planned behavior. Engineering Management, IEEE, [S.I], v. 52, n. 1, p. 69-84, 2005.

MUSCANELL, N.L.; GUADAGNO, R. E. Make new friends or keep the old: Gender and personality differences in social networking use. Computers in Human Behavior, [S.I],v. 28, n. 1, p. 107-112, 2012.

NASCIMENTO JÚNIOR, N.; PIMENTEL, E. P.; DOTTA, S.; BRAGA, J. C. Gerenciamento do Acompanhamento de Atividades Acadêmicas em uma disciplina conduzida através da Rede Social Facebook. Conferencias LACLO, [S.I], v. 4, n. 1, 2013.

NOSKO, A.; WOOD,E.; MOLEMA, S. All about me: Disclousure in online social networking profiles: The case of Facebook. Computers in Human Behavior, [S.I], v. 26, n. 3, p. 406-418, 2010.

OLIVEIRA, M. J.; HUERTAS, M. K. Z. A satisfação com a vida influencia a intenção de uso do Facebook? REMark - Revista Brasileira de Marketing, [S.I], v. 13, n. 6, p. 124-137, Out./Dez. 2014.

OMAR, M. K.; DAHALAN, N. A.; YUSOFF, Y. H. M. Social Media Usage, Perceived Team-Efficacy and knowledge Sharing Behavior among Employees of an Oil and Gas Organization in Malaysia. Procedia Economics and Finance, [S.I],v. 37, n. 1, p. 309-316, 2016.

PANTANO, E.; GANDINI, A. Exploring the forms of sociality mediated by innovative technologies in retail settings. Computers in Human Behavior, [S.I],v. 3, n. 1, p.1-7, 2017.

PARK, N.; LEE, S.; KIM, J.H. Individuals personal characteristics and patterns of Facebook use: A social network approach. Computers in Human Behavior, [S.I],v. 28, n. 1, p. 1700-1707, 2012. 
PESTANA, M. H.; GAGEIRO, J. N. Análise de dados para ciências sociais: a complementaridade do SPSS. Lisboa: Silabo, 2003.

PFEIL, U. R. ARJAN, P. Zaphiris. Age differences in online social networking: A study of user profiles and the social capital divide among teenagers and older users in MySpace. Computers in Human Behavior, [S.I],v. 25, n. 3, p. 643-654, 2009.

ROCHA, R. R. ; CHRISTOPOULOS, T. P. Da possibilidade de exploração do capital social no mundo virtual: um caleidoscópio do twitter e os candidatos à prefeitura de São Paulo. In: ENCONTRO DA ADMINISTRAÇÃO DA INFORMAÇÃO, 4., 2013, Bento Gonçalves. Anais... Bento Gonçalves: EnADI, 2013. p. 1-16.

RYAN, T.; XENOS, S. Who uses facebook? An investigation into the relationship between the big five, shyness, narcissism, loneliness, and facebook usage. Computers in Human Behavior, [S.I], v. 27, n. 5 , set. p. 1658-1664, 2011.

SEEBALUCK, N. V.; BHIWAJEE, S. D. L.; RAMSEOOK-MUNHURRUN, P. R.; DABEEDIN, Y. Assessing the Saturation Potential of Social Networks: A Mauritian Perspective. Procedia- Social and Behavioral Schiences. [S.I], v.175, n. 1, p. 193-200, 2015.

SHELDON, P. Understanding students reasons and gender differences in adding faculty as Facebook friends. Computers in Human Behavior, [S.I], v. 53, n. 1, p. 58-62, 2015.

SHIMAZAKI, V. K.; PINTO, M. M. M. A influência das redes sociais na rotina dos seres humanos. Disponível em: <http://www.fatecsaocaetano.edu.br/fascitech/index.php/fascitech/article/view/57/56>. Acesso em: 16 abril 2014.

SLEDGIANOWSKI, D.; KULVIWAT, S. Using Social Network Sites: the effects of playfulness, critical mass and trust in a hedonic context. Journal of Computer Information Systems, [S.I], v. 49, n. 4, p. 7483, 2009.

SOUZA, F. M.; FILENGA, D.; SANCHEZ, O. P. O impacto da influência social sobre a intenção de uso de sites de compras coletivas: um estudo baseado no modelo UTAUT, com usuários do Orkut, Facebook, Twitter e Linkedin. In: ENCONTRO DA ADMINISTRAÇÃO DA INFORMAÇÃO, 3., 2011, Porto Alegre. Anais... Porto Alegre: EnADI, 2011. p.1-13.

SUN, Y. et al. Location information disclosure in location-based social network services: Privacy calculus, benefict structure, and gender differences. Computers in Human Behavior, [S.I], v. 52, n. 1, p. 278-292, 2015.

SZAJNA, B. Empirical Evaluation of the Revised Technology Acceptance Model. Management Science, [S.I], v. 42, n. 1, p. 85-92. 1996.

TAPSCOTT, D. Geração digita: a crescente e irreversível ascensão da Geração Net. São Paulo: Makron Books, 1999.

TARHINI, A.; HONE, K.; LIU, X. Measuring the moderating effect of gender and age on e-learning acceptance in England: a structural equation modeling approach for an extended technology acceptance model. Journal Educational Computing Research, [S.I], v. 51, n. 2, p. 163-184, 2014.

TRIVIÑOS, A. N.S. Introdução à pesquisa em ciências sociais: a pesquisa qualitativa em educação. São Paulo: Atlas, 2007. 175 p.

VENKATESH, V. et al. User Acceptance of Information Technology: Toward a Unified View. MIS Quarterly, [S.I], v. 27, n. 3, p. 425-478, 2003.

VENKATESH, V.; MORRIS, M.G. Why don't men ever stop to ask for directions? Gender, social influence, and their role in technology acceptance and usage behavior. Mis Quarterly, [S.I], v. 24, n. 1, p. 115-139, 2000. 
VENKATESH, V.; THONG, J.Y.L.; XU, X. Consumer Acceptance and Use of Information Technology: Extending the Unified Theory of Acceptance and Uso of Technology.MIS Quarterly, [S.I], v. 36, n.1, 2012. VERHAGEN, T.; FELDBERG, F.; van den HOOFF, B.; MEENTS, S.; MERIKIVI, J. Understanding users' motivations to engage in virtual worlds: A multipurpose model and empirical testing. Computers in Human Behavior, [S.I], v. 28, n. 2, p. 484-495, 2012.

VISENTINI, M. S. et al. Curte, comenta ou compartilha? Investigando fatores que influenciam a intenção comportamental de uso do Facebook. Espacios, [S.I], v. 37, n. 11, p. 1-16, 2016.

ZAGO, G. S.; BASTOS, M. T. Visibilidade de notícias no Twitter e no Facebook: Análise comparativa das notícias mais repercutidas na Europa e nas Américas. Brazilian Journalism Research, [S.I], v. 9, n. 1, p. 116-133, 2013.

Submetido em: 24/02/2017

Aprovado em: 13/06/2017 